Network Working Group

Request for Comments: 4530

Category: Standards Track
K. Zeilenga

OpenLDAP Foundation

June 2006

\title{
Lightweight Directory Access Protocol (LDAP) entryUUID Operational Attribute
}

Status of This Memo

This document specifies an Internet standards track protocol for the Internet community, and requests discussion and suggestions for improvements. Please refer to the current edition of the "Internet Official Protocol Standards" (STD 1) for the standardization state and status of this protocol. Distribution of this memo is unlimited.

Copyright Notice

Copyright (C) The Internet Society (2006).

Abstract

This document describes the LDAP/X.500 'entryUUID' operational attribute and associated matching rules and syntax. The attribute holds a server-assigned Universally Unique Identifier (UUID) for the object. Directory clients may use this attribute to distinguish objects identified by a distinguished name or to locate an object after renaming. 
Table of Contents

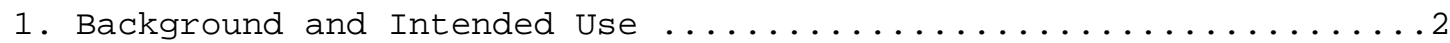

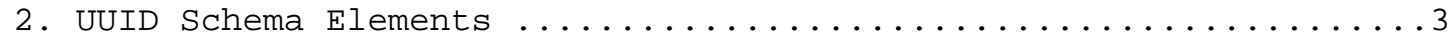

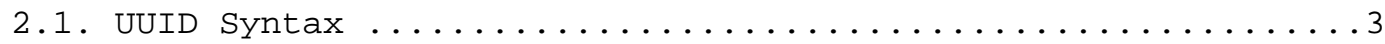

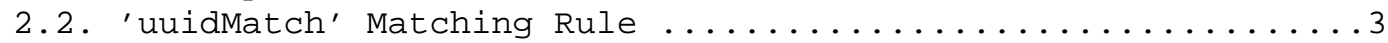

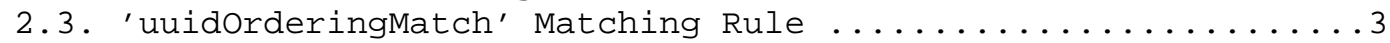

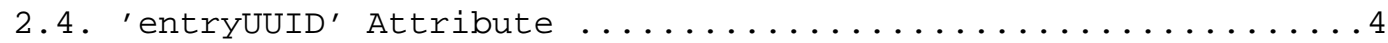

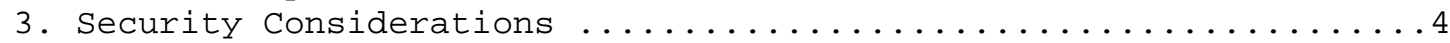

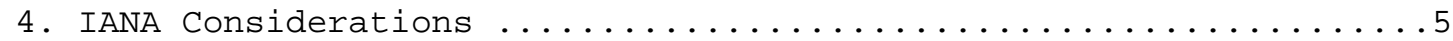

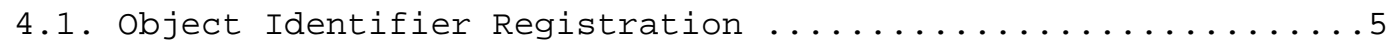

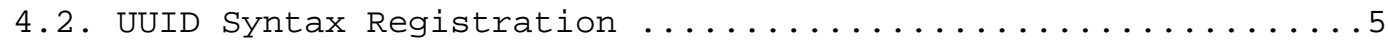

4.3. 'uuidMatch' Descriptor Registration ...............

4.4.' 'uuidOrderingMatch' Descriptor Registration ..........5

4.5. 'entryuUID' Descriptor Registration ............... 6

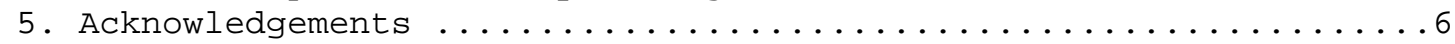

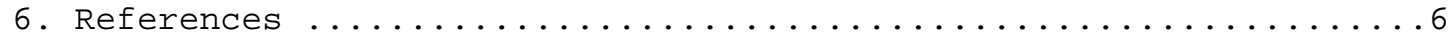

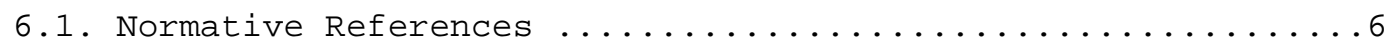

6.2. Informative References .......................

1. Background and Intended Use

In X.500 Directory Services [X.501], such as those accessible using the Lightweight Directory Access Protocol (LDAP) [RFC4510], an object is identified by its distinguished name (DN). However, DNs are not stable identifiers. That is, a new object may be identified by a DN that previously identified another (now renamed or deleted) object.

A Universally Unique Identifier (UUID) is "an identifier unique across both space and time, with respect to the space of all UUIDs" [RFC4122]. UUIDs are used in a wide range of systems.

This document describes the 'entryUUID' operational attribute, which holds the UUID assigned to the object by the server. Clients may use this attribute to distinguish objects identified by a particular distinguished name or to locate a particular object after renaming.

This document defines the UUID syntax, the 'uuidMatch' and ' uuidOrderingMatch' matching rules, and the 'entryUUID' attribute type.

Schema definitions are provided using LDAP description formats [RFC4512]. Definitions provided here are formatted (line wrapped) for readability. 
In this document, the key words "MUST", "MUST NOT", "REQUIRED", "SHALL", "SHALL NOT", "SHOULD", "SHOULD NOT", "RECOMMENDED", "MAY", and "OPTIONAL" are to be interpreted as described in BCP 14 [RFC2119].

2. UUID Schema Elements

2.1. UUID syntax

A Universally Unique Identifier (UUID) [RFC4122] is a 16-octet (128bit) value that identifies an object. The ASN.1 [X.680] type UUID is defined to represent UUIDs as follows:

UUID : := OCTET STRING (SIZE(16)) -- constrained to an UUID [RFC4122]

In LDAP, UUID values are encoded using the [ASCII] character string representation described in [RFC4122]. For example, "597ae2f6-16a6-1027-98f4-d28b5365dc14".

The following is an LDAP syntax description suitable for publication in subschema subentries.

$$
\text { ( } 1.3 .6 .1 .1 .16 .1 \mathrm{DESC} \text { 'UUID' ) }
$$

\section{2. 'uuidMatch' Matching Rule}

The 'uuidMatch' matching rule compares an asserted UUID with a stored UUID for equality. Its semantics are the same as the 'octetstringMatch' [X.520][RFC4517] matching rule. The rule differs from 'octetStringMatch' in that the assertion value is encoded using the UUID string representation instead of the normal OCTET STRING string representation.

The following is an LDAP matching rule description suitable for publication in subschema subentries.

( 1.3.6.1.1.16.2 NAME 'uuidMatch' SYNTAX 1.3.6.1.1.16.1)

\section{3. 'uuidOrderingMatch' Matching Rule}

The 'uuidorderingMatch' matching rule compares an asserted UUID with a stored UUID for ordering. Its semantics are the same as the 'octetstringOrderingMatch' [X.520] [RFC4517] matching rule. The rule differs from 'octetStringorderingMatch' in that the assertion value is encoded using the UUID string representation instead of the normal OCTET STRING string representation. 
The following is an LDAP matching rule description suitable for publication in subschema subentries.

( 1.3.6.1.1.16.3 NAME 'uuidOrderingMatch' SYNTAX 1.3.6.1.1.16.1)

Note that not all UUID variants have a defined ordering; and even where it does, servers are not obligated to assign UUIDs in any particular order. This matching rule is provided for completeness.

\section{4. 'entryUUID' Attribute}

The 'entryUUID' operational attribute provides the Universally Unique Identifier (UUID) assigned to the entry.

The following is an LDAP attribute type description suitable for publication in subschema subentries.

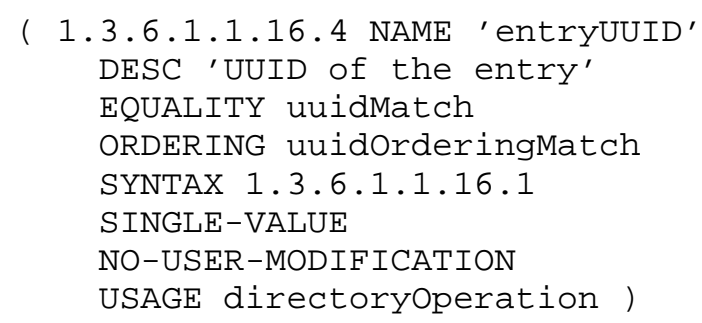

Servers SHALL generate and assign a new UUID to each entry upon its addition to the directory and provide that UUID as the value of the 'entryUUID' operational attribute. An entry's UUID is immutable.

UUID are to be generated in accordance with section 4 of [RFC4122]. In particular, servers MUST ensure that each generated UUID is unique in space and time.

3. Security Considerations

An entry's relative distinguish name (RDN) is composed from attribute values of the entry, which are commonly descriptive of the object the entry represents. Although deployers are encouraged to use naming attributes whose values are widely disclosable [RFC4514], entries are often named using information that cannot be disclosed to all

parties. As UUIDs do not contain any descriptive information of the object they identify, UUIDs may be used to identify a particular entry without disclosure of its contents.

General UUID security considerations [RFC4122] apply. 
General LDAP security considerations [RFC4510] apply.

4. IANA Considerations

The IANA has registered the LDAP values [RFC4520] specified in this document.

4.1. Object Identifier Registration

Subject: Request for LDAP OID Registration

Person \& email address to contact for further information:

Kurt Zeilenga <kurt@openLDAP.org>

Specification: RFC 4530

Author/Change Controller: IESG

Comments:

Identifies the UUID schema elements

4.2. UUID Syntax Registration

Subject: Request for LDAP Syntax Registration

Object Identifier: 1.3.6.1.1.16.1

Description: UUID

Person \& email address to contact for further information:

Kurt Zeilenga <kurt@openLDAP.org>

Specification: RFC 4530

Author/Change Controller: IESG

Comments :

Identifies the UUID syntax

4.3. 'uuidMatch' Descriptor Registration

Subject: Request for LDAP Descriptor Registration

Descriptor (short name): uuidMatch

Object Identifier: 1.3.6.1.1.16.2

Person \& email address to contact for further information:

Kurt Zeilenga <kurt@openLDAP.org>

Usage: Matching Rule

Specification: RFC 4530

Author/Change Controller: IESG

4.4. 'uuidOrderingMatch' Descriptor Registration

Subject: Request for LDAP Descriptor Registration

Descriptor (short name): uuidorderingMatch

Object Identifier: 1.3.6.1.1.16.3

Person \& email address to contact for further information:

Kurt Zeilenga <kurt@openLDAP.org>

Usage: Matching Rule 


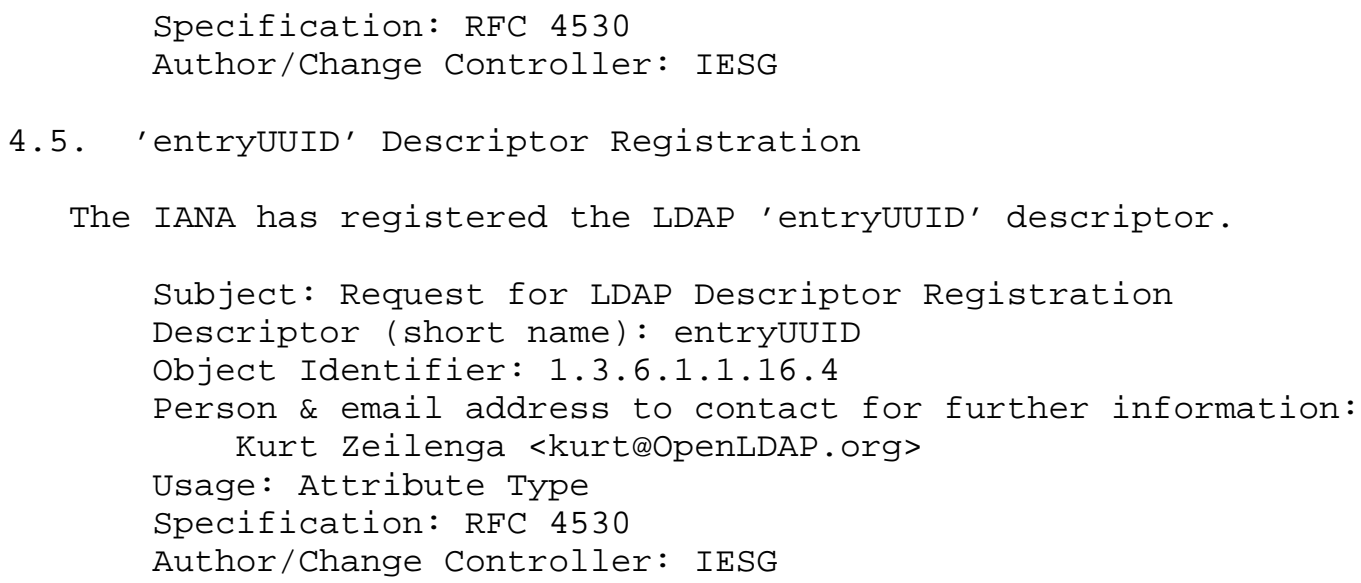

5. Acknowledgements

This document is based upon discussions in the LDAP Update and Duplication Protocols (LDUP) WG. Members of the LDAP Directorate provided review.

6. References

6.1. Normative References

[RFC2119] Bradner, S., "Key words for use in RFCs to Indicate Requirement Levels", BCP 14, RFC 2119, March 1997.

[RFC4122] Leach, P., Mealling, M., and R. Salz, "A Universally Unique IDentifier (UUID) URN Namespace", RFC 4122, July 2005 .

[RFC4510] Zeilenga, K., Ed., "Lightweight Directory Access Protocol (LDAP): Technical Specification Road Map", RFC 4510, June 2006 .

[RFC4512] Zeilenga, K., "Lightweight Directory Access Protocol (LDAP): Directory Information Models", RFC 4512, June 2006 .

[RFC4517] Legg, S., Ed., "Lightweight Directory Access Protocol (LDAP): Syntaxes and Matching Rules", RFC 4517, June 2006 .

[ASCII] Coded Character Set--7-bit American Standard Code for Information Interchange, ANSI X3.4-1986. 
[X.501] International Telecommunication Union Telecommunication Standardization Sector, "The Directory -- Models," X.501(1993) (also ISO/IEC 9594$2: 1994)$.

[X.520] International Telecommunication Union Telecommunication Standardization Sector, "The Directory: Selected Attribute Types", X.520(1993) (also ISO/IEC 9594-6:1994).

[X.680] International Telecommunication Union Telecommunication Standardization Sector, "Abstract Syntax Notation One (ASN.1) - Specification of Basic Notation", X.680(2002) (also ISO/IEC 8824-1:2002).

6.2. Informative References

[RFC4514] Zeilenga, K., Ed., "Lightweight Directory Access Protocol (LDAP): String Representation of Distinguished Names", RFC 4514, June 2006.

[RFC4520] Zeilenga, K., "Internet Assigned Numbers Authority (IANA) Considerations for the Lightweight Directory Access Protocol (LDAP)", BCP 64, RFC 4520, June 2006.

Author's Address

Kurt D. Zeilenga

OpenLDAP Foundation

EMail: Kurt@openLDAP.org 
Full Copyright statement

Copyright (C) The Internet Society (2006).

This document is subject to the rights, licenses and restrictions contained in BCP 78, and except as set forth therein, the authors retain all their rights.

This document and the information contained herein are provided on an "AS IS" basis and THE CONTRIBUTOR, THE ORGANIZATION HE/SHE REPRESENTS OR IS SPONSORED BY (IF ANY), THE INTERNET SOCIETY AND THE INTERNET ENGINEERING TASK FORCE DISCLAIM ALL WARRANTIES, EXPRESS OR IMPLIED, INCLUDING BUT NOT LIMITED TO ANY WARRANTY THAT THE USE OF THE INFORMATION HEREIN WILL NOT INFRINGE ANY RIGHTS OR ANY IMPLIED WARRANTIES OF MERCHANTABILITY OR FITNESS FOR A PARTICULAR PURPOSE.

Intellectual Property

The IETF takes no position regarding the validity or scope of any Intellectual Property Rights or other rights that might be claimed to pertain to the implementation or use of the technology described in this document or the extent to which any license under such rights might or might not be available; nor does it represent that it has made any independent effort to identify any such rights. Information on the procedures with respect to rights in RFC documents can be found in BCP 78 and BCP 79 .

Copies of IPR disclosures made to the IETF Secretariat and any assurances of licenses to be made available, or the result of an attempt made to obtain a general license or permission for the use of such proprietary rights by implementers or users of this specification can be obtained from the IETF on-line IPR repository at http://www.ietf.org/ipr.

The IETF invites any interested party to bring to its attention any copyrights, patents or patent applications, or other proprietary rights that may cover technology that may be required to implement this standard. Please address the information to the IETF at ietf-ipreietf.org.

Acknowledgement

Funding for the RFC Editor function is provided by the IETF Administrative Support Activity (IASA). 